\title{
REDUCTION OF STIMULATED BRILLOUIN SCATTERING THRESHOLD USING BIDIRECTIONAL PUMPING WITH PUMP RECYCLING SCHEME
}

\author{
M.H. Al-Mansoori ${ }^{1}{ }^{*}$ F. Al-Rubai ${ }^{2}$ and A. Al-Jabri ${ }^{3}$
}

\begin{abstract}
In this paper, a reduction of stimulated Brillouin scattering (SBS) threshold using bidirectional pumping with pump recycling technique is demonstrated. Two pumping schemes; direct method, and bidirectional pumping with recycling method are experimentally investigated. Using $10 \mathrm{~km}$ single mode fiber, the proposed technique exhibits a low threshold power of $6 \mathrm{~mW}$ against $9.5 \mathrm{~mW}$ for direct pumping method. In addition, the measured Stokes signal power is higher for bidirectional pumping with recycling scheme. The results have proved that the proposed technique improved the Stokes signal power and reduced the SBS threshold power compared to direct injection method.
\end{abstract}

Keywords - Optical fiber; Stimulated Brillouin scattering; Threshold power

\section{INTRODUCTION}

Optical communication system use optical fiber as a transmission medium which can carry an enormous amount of information between transmitters and receivers. The light forms an electromagnetic carrier wave that is modulated to carry information [1]. Optical fiber has issues depend on the level of the signal power transmitted through it called nonlinear characteristic. Stimulated Brillouin scattering (SBS) is one of the dominant nonlinear effects in optical fiber. The first demonstration of SBS was predicted by L. Brillouin in 1922 [2]. Then, the stimulated process, when the acoustic wave is created was observed by Chiao et.al [3]. The SBS was observed by employing low-loss mono-fiber at low power levels and narrowband laser transmitter in 1983 [4]. Moreover, K.S. Abedin investigated the strong SBS in single mode As2Se3 chalcogenide fiber by using a $\mathrm{CW}$ laser at $1.55 \mu \mathrm{m}$ wavelength regions, which found a threshold power of $85 \mathrm{~mW}$ [5]. Various techniques were used for characterizing the SBS effect in optical fiber [6-10]. Reduction of SBS threshold in the existence of the counter propagating pump in a low loss long fiber of $12.5 \mathrm{~km}$ was demonstrated in [6]. The reduction on the Brillouin threshold attributed to the coupling between the forward and backward Stokes signals.

Two tunable laser sources were used by A. Villafranca et.al [7] to investigate the SBS process in optical fiber. One of the lasers was used as probe signal and the other one as a pump signal. A high signal to noise ratio SBS gain profile and the applications of SBS for filtering were

${ }^{1}$ Electrical and Computer Engineering, Faculty of Engineering, Sohar University, Sohar, PO Box 44, PC 311, Sultanate of Oman

${ }^{2}$ Electrical and Computer Engineering, Faculty of Engineering, Sohar University, Sohar, PO Box 44, PC 311, Sultanate of Oman

${ }^{3}$ Electrical and Computer Engineering, Faculty of Engineering, Sohar University, Sohar, PO Box 44, PC 311, Sultanate of Oman 
demonstrated. A high threshold power of $1 \mathrm{~W}$ at $2 \mathrm{~km}$ length fiber was reported. The study of SBS threshold using different refractive index profiles of the fiber was reported by A. Kobyakov et.al, [8]. They reported that the acousto-optic effective area that quantifies the SBS depends on the radial distribution of the core dopant concentration and a large acousto-optic effective area is needed to reduce the Brillouin threshold. A threshold power of $11.5 \mathrm{dBm}$ for a fiber length of 20 $\mathrm{km}$ with an effective area of $85.4 \mu \mathrm{m} 2$ was obtained. In addition, T. Shimizu et.al reported different methods to evaluate the SBS threshold in term of harmonization with analogue transmission characteristics [9]. The researchers used three type of single mode fiber with effective area of $78.2 \mu \mathrm{m} 2,80.9 \mu \mathrm{m} 2$ and $76.1 \mu \mathrm{m} 2$ at a length of $25 \mathrm{~km}$. The condition to find power threshold was investigated. A theoretical analysis to estimate the SBS threshold power with pump recycling technique was developed by H.A. Al-Asadi et.al [10]. An optical mirror was used at the other fiber end to reflect the transmitted signal back to the Brillouin gain medium. The pump recycling technique reduced the threshold power from $16 \mathrm{~mW}$ using conventional method to about $8 \mathrm{~mW}$ in a $5 \mathrm{~km}$ fiber length. Recently, a comprehensive theoretical study of SBS threshold power in single mode fiber was reported by R. Parvizi [11]. The pump depletion effect and fiber loss was included in the theoretical analysis. The calculated threshold power for $30 \mathrm{~km}$ fiber length with effective area of $50 \mu \mathrm{m}^{\wedge} 2$ was found equal to 1.1 $\mathrm{mW}$. Although SBS phenomenon reduces the received signal power, because part of the transmitted signal reflected back due to the material density and compositional fluctuations [12]. This can be considered as negative features. However, this can be utilized as a positive purpose by using it in some applications like fiber sensors, fiber lasers and amplifiers. Therefore, a reduction in SBS threshold is needed for efficient utilization of this nonlinear effect for several applications. In addition, no study has been conducted to show the influence of bidirectional pumping with recycling of transmitted signal on the SBS threshold reduction and Stokes signal power.

In this paper, we experimentally demonstrate a simple technique to reduce the Brillouin threshold power using bidirectional pumping with pump recycling method. Using $10 \mathrm{~km}$ single mode fiber, the proposed technique exhibits a low threshold power of $6 \mathrm{~mW}$ against $9.5 \mathrm{~mW}$ for direct pumping method. In addition, the proposed technique has higher Stokes signal power as compared to direct injection technique.

\section{EXPERIMENTAL SETUP}

Figure 1 depicts the experimental setup of SBS characterization using bidirectional pumping scheme with pump recycling method. The structure that contains tunable laser source (TLS) to provide the input signal (Brillouin pump). Variable optical coupler is used, where the output ports named as coupled port (CP) and throughput port (TP) are connected to the fiber ends through an optical circulator and a $3 \mathrm{~dB}$ coupler. The TP is connected to port 1 of the circulator and the $\mathrm{CP}$ is connected to the output port of the $3 \mathrm{~dB}$ coupler. An optical mirror is connected to the other port of the coupler to reflect the transmitted pump signal. A length of $10 \mathrm{~km}$ of single mode fiber (SMF) is used as a gain medium. The first end of the SMF is connected to port 2 of the circulator while the other end is connected to the input port of the $3 \mathrm{~dB}$ coupler. The input signal will enter the variable optical coupler first, and then it will be guided into both ends of the SMF through the $3 \mathrm{~dB}$ coupler and optical circulator. When the input signal power exceeds the Brillouin threshold power of the SMF, the first order Stokes signal will be created and propagate in the opposite direction to the direction of the input signal. The Stokes signal power is measured 
by an optical spectrum analyser (OSA) through port 3 of the optical circulator as depicted on Figure 1.

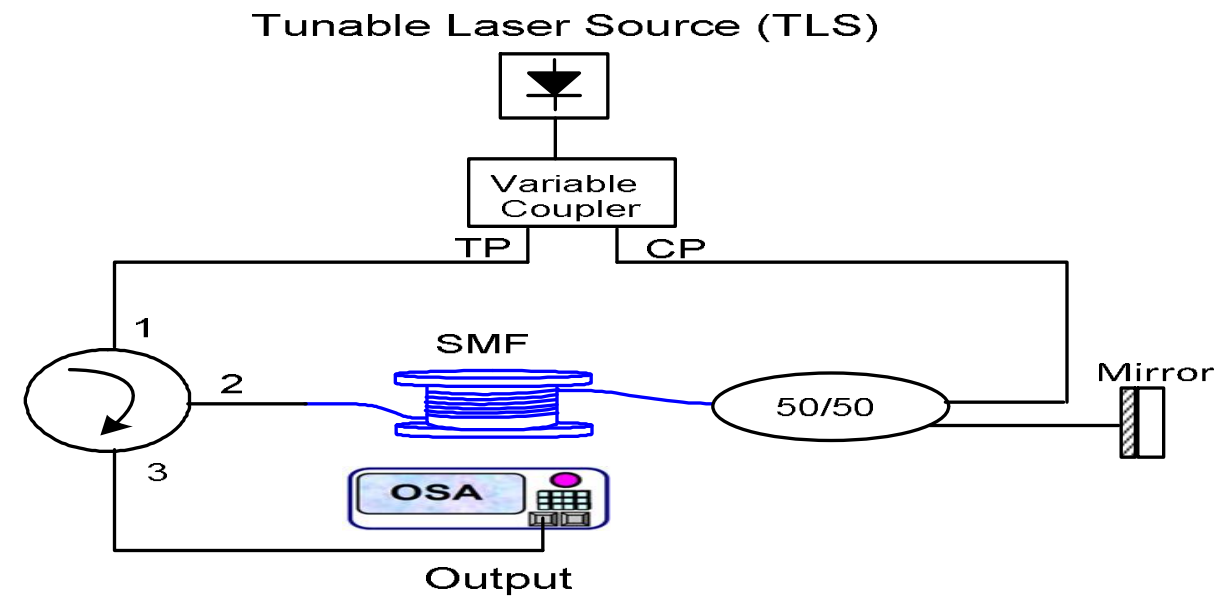

Figure 1: The structure of SBS characterization using bidirectional-pumping scheme with pump recycling method

\section{RESULTS AND DISCUSSION}

Scattering of light requires high incident light to be scattered from the acoustic waves. At low input power there is no back scattering (Stokes signal) signal. Once the input signal power exceeds the Brillouin threshold level, most of the signal power reflected backward. Figure 2 shows the relation between the Stokes signal powers with the input signal power for bidirectional pumping with recycling of pump technique. A lowest threshold power is founded in the bidirectional pumping with recycling the transmitted power which equal to $6 \mathrm{~mW}$ against 9.5 $\mathrm{mW}$ for direct pumping method (conventional method of SBS characterization). In addition, at input signal power of $23 \mathrm{~mW}$, the measured Stokes signal power was $8.6 \mathrm{~mW}$ for direct injection and $11.6 \mathrm{~mW}$ for bidirectional pumping with recycling method. The bidirectional pumping with recycling method provide better Stokes signal output power. 


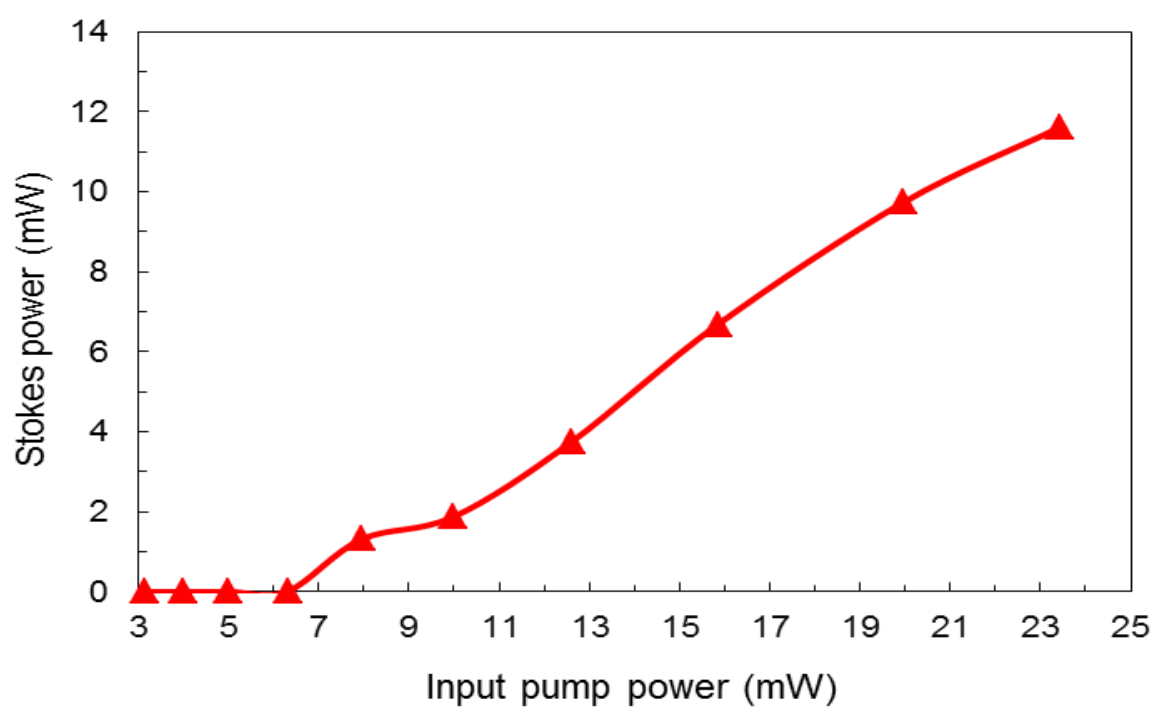

Figure 2: Stokes signal power with the input signal power for direct injection and bidirectional pumping with recycling method

Add an optical mirror in direct injection method change the method to recycling the transmitted signal back into the SMF. The optical mirror allow more backscattered light which can be reduce the level of the threshold power and speed up the appearance of the SBS [13-14]. For the purpose of investigating the effect of input coupling ratio between the direct pumping and proposed methods, a variable coupler with different coupling ratio is used to distribute the input signal. The input signal power is varied from $1 \mathrm{~mW}$ to $23 \mathrm{~mW}$ for coupling ratio of 50/50, 70/30 and 90/10. Figure 3 shows the effect of coupling ratio of the input signal on the Stokes signal power and the threshold power. It is clear that the threshold power has different value for different coupling ratio. When the coupling ratio is adjusted, there is a balance for the distribution of the signal that passes through the TP and CP ports. The 50/50 coupling ratio means that TP will take 50 and $\mathrm{CP}$ will takes 50 from the input signal power. Stokes signal power depends on the input signal power that comes from TP port, TC port and the recycling of the transmitted pump signal. By increasing the input signal power, the Stokes signal power increases. For 50/50 input coupling ratio, the threshold power recorded are about $23 \mathrm{~mW}, 10.5 \mathrm{~mW}$ for direct injection method and bidirectional pumping with recycling method, respectively. The threshold power is reduced from $23 \mathrm{~mW}$ to $10.5 \mathrm{~mW}$ when bidirectional pumping with recycling method is used. In addition at $90 / 10$ coupling ratio, $90 \%$ of the input signal passes to TP port and $10 \%$ passes to $\mathrm{CP}$. Adjusting the rate of the coupling ratio to level that allow more signal to pass through the $\mathrm{CP}$ port helps to increase the amount of the back scattering signal and reduces the threshold power. For $90 / 10$ coupling ratio, the measured threshold power is about $8 \mathrm{~mW}$ for bidirectional pumping with recycling method. 

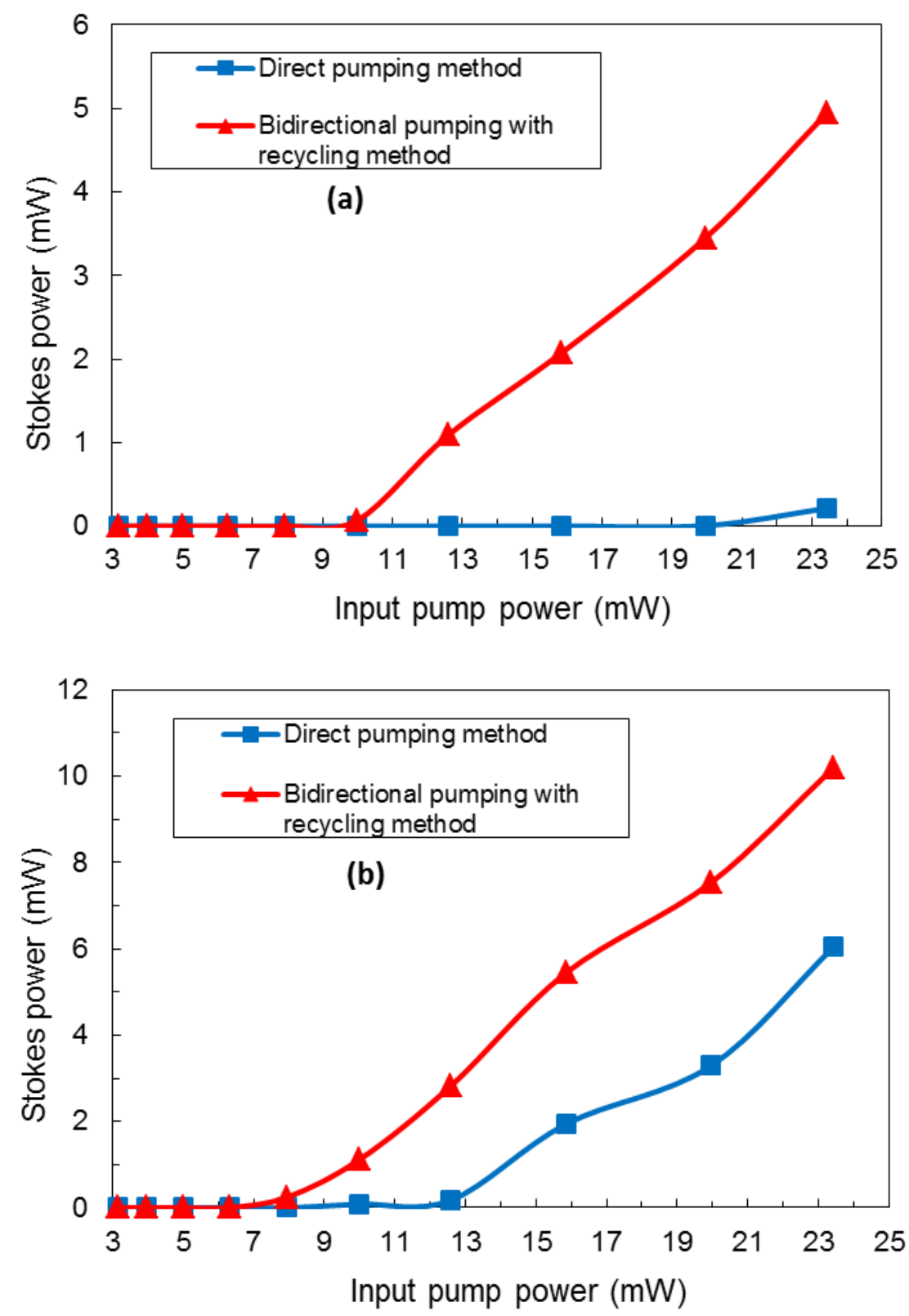

Figure 3: Stokes signal power with the input signal power for direct injection and bidirectional pumping with recycling method for different coupling ratio of (a) 50/50 and (b) 90/10

The proposed method enhanced the Stokes signal power for different coupling ration. Figure 4 shows the Stokes signal power at different input coupling ratio for bidirectional pumping with recycling method. After the threshold power, the Stokes signal power increases with the increase on the input signal power and coupling ratio. For 70/30 coupling ratio and input power of 20 $\mathrm{mW}$, the Stokes signal power increases from $3.3 \mathrm{~mW}$ to $7.5 \mathrm{~mW}$ for the direct injection of the pump and the proposed methods, respectively. This is attributed to the additional energies provided by the bidirectional pumping and recycled pump signal which helps to initiate more stimulated Brillouin scattering emissions and translate that to additional amplification for the 
Stokes signal. The effect of fiber length on the Stokes signal power and threshold power is also investigated. Figure 5 shows the measured Stokes signal power with respect to the input power at fiber length of $12.1 \mathrm{~km}$ and $10 \mathrm{~km}$. No Stokes signal appears unless the input signal power exceeds the Brillouin threshold level. For $12 \mathrm{~km}$ of SMF, the Stokes starts to appear when the input power is $5 \mathrm{~mW}$; it has peak power of $0.434 \mathrm{~mW}$. Low threshold power with high Stokes signal power is observed at longer SMF length. The Stokes signal power increase exponentially after the threshold power. At input signal power of $16 \mathrm{~mW}$, the measured Stokes signal power is $6.7 \mathrm{~mW}$ at $10 \mathrm{~km}$ and $7.8 \mathrm{~mW}$ at $12.1 \mathrm{~km}$ length of SMF. Furthermore, at input power of 23 $\mathrm{mW}$, the maximum measured Stokes signal power was $11.6 \mathrm{~mW}$ at $10 \mathrm{~km}$ and $12.8 \mathrm{~mW}$ at 12.1 $\mathrm{km}$. The threshold obtained for fiber length of $10 \mathrm{~km}$ and $12.1 \mathrm{~km}$ is approximately $6 \mathrm{~mW}$ and 5 $\mathrm{mW}$, respectively. The threshold is inversely varies with the length of the fiber.

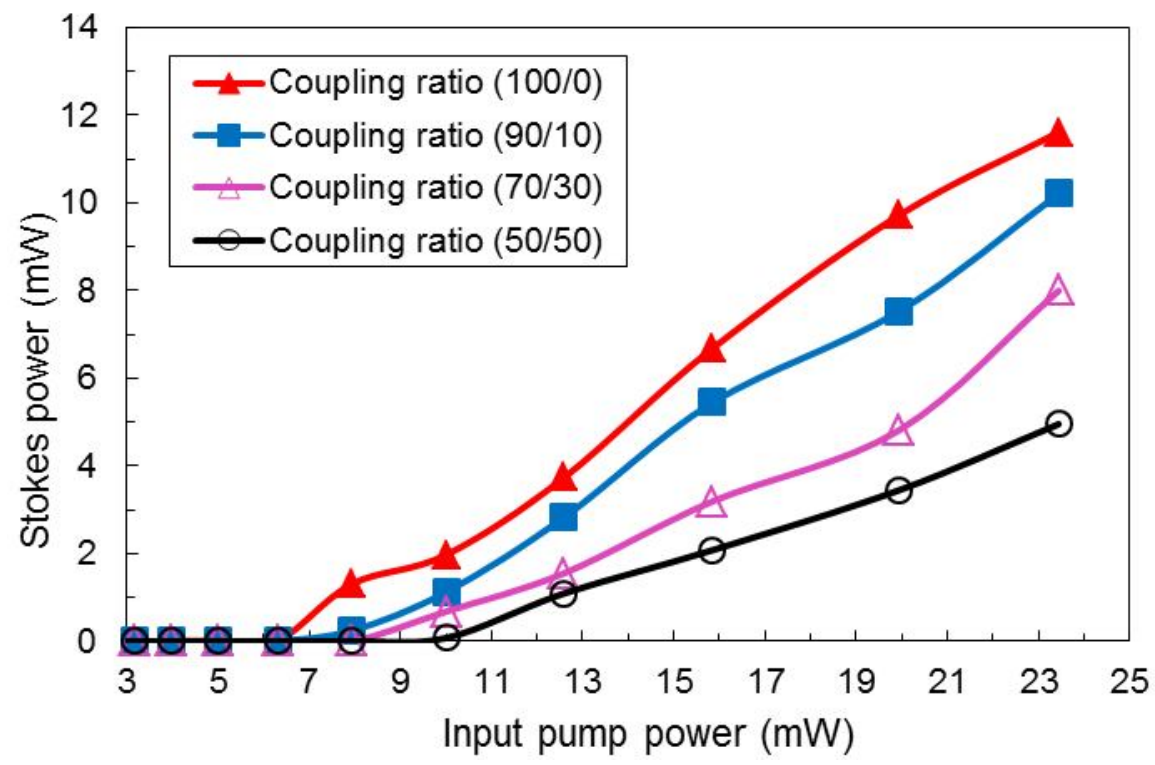

Figure 4: Stokes signal power verse input signal power at different input coupling ratio for bidirectional pumping with recycling method 


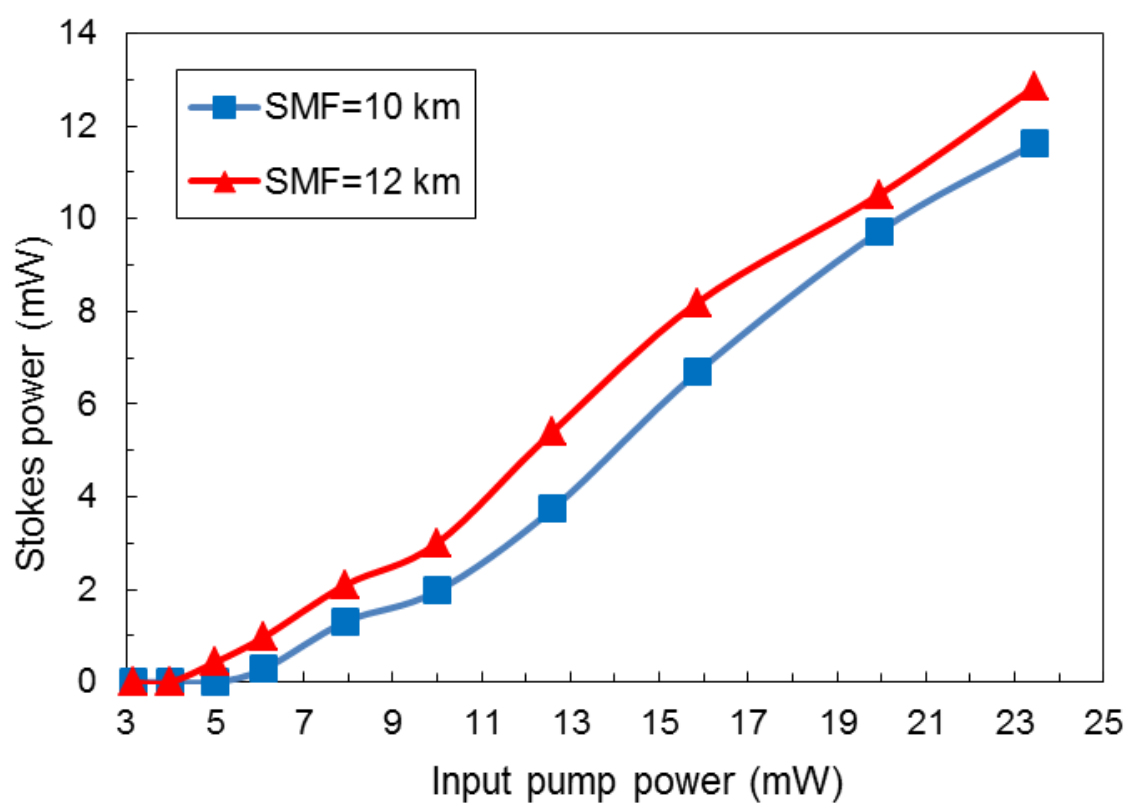

Figure 5: Stokes signal power verse input signal power at different SMF lengths of $10 \mathrm{~km}$ and $12.1 \mathrm{~km}$

\section{IV.CONCLUSION}

A simple technique to reduce the stimulated Brillouin scattering threshold and increase the Stokes signal power has successfully demonstrated. We utilized a bidirectional pumping scheme with pump recycling method using high reflectivity optical mirror to increase the Brillouin gain efficiency in optical fiber. The proposed technique solved the issue of low gain efficiency of Brillouin gain and produce high Stokes signal power. In addition, it exhibits a low threshold power with maximum Stokes signal power. Moreover, the use of variable coupling ratio for the input signal produces a lowest threshold power. The proposed technique has provided advantage in increasing the Stokes signal power and reducing the SBS threshold power which can be utilized to generate and efficient multiwavelength Brillouin fiber laser.

\section{ACKNOWLEDGMENT}

The research leading to these results has received research project grant funding from the Research Council of the Sultanate of Oman under Research Grant Agreement No. [ORG SU ICT 11 002]. The authors also acknowledge the support from Sohar University, Oman.

\section{REFERENCES}

[1] J.M. Senior, "Optical Fiber Communications Principles and Practice," third Edition, Prentice Hall, London, 2009.

[2] G.P. Agrawal, "Fiber-Optic Communication Systems," 4th Edition, Academic Press, New York, University of Rochester, 2007.

[3] R.Y. Chiao, C.H. Townes, and B.P. Stoicheff, "Stimulated Brillouin Scattering and Coherent Generation of Intense Hypersonic Waves", Physical review letters, Vol. 12, no. 21, pp. 592-595, 1964. 
[4] D. Cotter, "Stimulated Brillouin scattering in monomode optical fiber," journal of optical commutations, Vol. 4, no. 1, pp. 10-19, 1983.

[5] K.S. Abedin, "Observation of strong stimulated Brillouin scattering in single-mode As2Se3 chalcogenide fiber", Optics Express, Vol. 13, no. 25, pp.10266-10271, 2005.

[6] K. Inoue, "Brillouin threshold in an optical fiber with bidirectional pump lights," Optics Communications, vol. 120, pp. 34-38, 1995.

[7] A. Villafranca, J.A. Lázaro, Í. Salinas and I. Garcés, "Stimulated Brillouin scattering gain profile characterization by interaction between two narrow-linewidth optical sources", Optics Express, Vol. 13, No. 19, pp. 7336- 7341, 2005.

[8] A. Kobyakov, S. Kumar, D.Q. Chowdhury, A.B. Ruffin, M. Sauer, S.R. Bickham and R. Mishra. "Design concept for optical fibers with enhanced SBS threshold", Optics Express, Vol. 13, No. 14, pp. 5338- 5346, 2005.

[9] T. Shimizu, K. Nakajima, K. Shiraki, K. Ieda and I. Sankawa.” Evaluation methods and requirements for the stimulated Brillouin scattering threshold in a single-mode fiber," Optical Fiber Technology, Vol.14, pp.10-15, 2008.

[10] H.A. Al-Asadi, M. H. Al-Mansoori, M. Ajiya, S. Hitam, M. I. Saripan, and M. A. Mahdi, "Effects of pump recycling technique on stimulated Brillouin scattering threshold: A theoretical model," Optics Express, Vol. 18, No. 21, pp. 22339- 22347, 2010.

[11] R. Parvizi, "Comprehensive Theoretical Study on Threshold Power of Stimulated Brillouin Scattering in Single-Mode Fibers", International Journal of Optics and Photonics (IJOP), Vol. 7, No. 1, Winter-Spring, 2013.

[12] Govind. P. Agrawal, Nonlinear Fiber Optics, 4th Edition, Rochester, New York: Elsevier, 2006, pg. 329367.

[13] M. Ajiya, M.A. Mahdi, M.H. Al-Mansoori, Y. G. Shee, S. Hitam and M. Mokhtar "Reduction of Stimulated Brillouin Scattering Threshold through Pump Recycling Technique," Laser Physics Letters, vol. 6, no. 3, pp. 535-538, 2009.

[14] Y. G. Shee, M.A. Mahdi, M.H. Al-Mansoori, A. Ismail, N.A.M. Hambali, A. K. Zamzuri, R. Mohamad, S. Yaakob, "Threshold Reduction of Stimulated Brillouin Scattering in Photonic Crystal Fiber," Laser Physics, vol. 19, no. 12, pp. 2194-2196, 2009. 